\title{
Innervation by a GABAergic Neuron Depresses Spontaneous Release in Glutamatergic Neurons and Unveils the Clamping Phenotype of Synaptotagmin-1
}

\author{
Keimpe D. B. Wierda ${ }^{1}$ and Jakob B. Sørensen ${ }^{1,2}$ \\ ${ }^{1}$ Neurosecretion Group, Signaling Laboratory, Department of Neuroscience and Pharmacology, University of Copenhagen, DK-2200 Copenhagen N, \\ Denmark, and '2Lundbeck Foundation Center for Biomembranes in Nanomedicine, University of Copenhagen, DK-2200 Copenhagen, Denmark
}

The role of spontaneously occurring release events in glutamatergic and GABAergic neurons and their regulation is intensely debated. To study the interdependence of glutamatergic and GABAergic spontaneous release, we compared reciprocally connected "mixed" glutamatergic/GABAergic neuronal pairs from mice cultured on astrocyte islands with "homotypic" glutamatergic or GABAergic pairs and autaptic neurons. We measured mEPSC and mIPSC frequencies simultaneously from both neurons. Neuronal pairs formed both interneuronal synaptic and autaptic connections indiscriminately. We find that whereas mEPSC and mIPSC frequencies did not deviate between autaptic and synaptic connections, the frequency of mEPSCs in mixed pairs was strongly depressed compared with either autaptic neurons or glutamatergic pairs. Simultaneous imaging of synapses, or comparison to evoked release amplitudes, showed that this decrease was not caused by fewer active synapses. The mEPSC frequency was negatively correlated with the mIPSC frequency, indicating interdependence. Moreover, the reduction in $\mathrm{MEPSC}$ frequency was abolished when established pairs were exposed to bicuculline for $3 \mathrm{~d}$, but not by long-term incubation with tetrodotoxin, indicating that spontaneous GABA release downregulates mEPSC frequency. Further investigations showed that knockout of synaptotagmin-1 did not affect mEPSC frequencies in either glutamatergic autaptic neurons or in glutamatergic pairs. However, in mixed glutamatergic/GABAergic pairs, mEPSC frequencies were increased by a factor of four in the synaptotagmin-1-null neurons, which is in line with data obtained from mixed cultures. The effect persisted after incubation with BAPTA-AM. We conclude that spontaneous GABA release exerts control over mEPSC release, and GABAergic innervation of glutamatergic neurons unveils the unclamping phenotype of the synaptotagmin-1-null neurons.

Key words: autaptic neuron; pHluorin imaging; spontaneous GABA release; spontaneous glutamate release; synaptotagmin-1

\section{Introduction}

Whereas action potential-evoked synaptic vesicle release is characterized by high temporal fidelity and speed, allowing reliable information transfer, spontaneous release by its very nature is unable to convey such time-locked information (Sutton and Schuman, 2009; Ramirez and Kavalali, 2011). Thus, the function of spontaneous release has been obscure, as such events might also result from random activation of the evoked release machinery. However, spontaneous release rates can be regulated independently of evoked release by activation of metabotropic glutamate receptors (Glitsch, 2006), and by transcriptional factors (Nelson et al., 2008), indicating that miniature release events

\footnotetext{
Received Sept. 13, 2013; revised Dec. 17, 2013; accepted Dec. 23, 2013.

Author contributions: K.D.B.W. and J.B.S. designed research; K.D.B.W. performed research; K.D.B.W. analyzed data; K.D.B.W. and J.B.S. wrote the paper.

This work was supported by the Lundbeck Foundation, the Novo Nordisk Foundation, the Danish Medical Research Council, and Grant HEALTH-F2-2009-242167 (SynSys Project; all to J.B.S.). K.D.B.W. was supported by an EMBO long-term fellowship. We thank A.M.N. Petersen for excellent technical assistance.

The authors declare no competing financial interests.

Correspondence should be addressed to Jakob B. Sørensen, Department of Neuroscience and Pharmacology, Faculty of Health Sciences, University of Copenhagen, Blegdamsvej 3B, 2200 Copenhagen N, Denmark. E-mail: jakobbs@sund.ku.dk.

DOI:10.1523/JNEUROSCI.3934-13.2014

Copyright $\odot 2014$ the authors $\quad 0270-6474 / 14 / 342100-11 \$ 15.00 / 0$
}

are sufficiently important to warrant separate regulation pathways. Indeed, specific functions for the spontaneous release of glutamate in regulating protein synthesis and stabilizing synapses have been described (Sutton et al., 2006, 2007). Furthermore, spontaneous release of single glutamate or GABA quanta suffice to affect postsynaptic firing in cerebellar stellate cells (Carter and Regehr, 2002). Finally, spontaneous release plays a role in reinforcing homeostatic plasticity mechanisms (Sutton et al., 2006, 2007; Aoto et al., 2008) and therefore might be important for setting overall network activity.

There is evidence that the properties of spontaneous glutamate release events deviate between autaptic neurons grown in isolation on astrocyte islands and neurons in mixed culture, or in brain slices. The most striking difference is the finding that deletion of synaptotagmin-1-the canonical calcium sensor for neurotransmitter release-is without consequence for mEPSC frequencies in autaptic neurons (Geppert et al., 1994; Liu et al., 2009), but leads to strong increases in mEPSC frequency in larger cultures or in brain slices (Broadie et al., 1994; Littleton et al., 1994; Pang et al., 2006; Chicka et al., 2008; Kerr et al., 2008; Kochubey and Schneggenburger, 2011). One reason for the discrepancy between systems might conceivably be the lack of GABAergic input experienced by glutamatergic neurons grown 
alone. Investigating astrocyte islands harboring more than one neuron makes it possible to systematically study how properties of synaptic transmission depend on the size of the network (Liu et al., 2009, 2013). Here, we investigated the hypothesis that reciprocal glutamatergic/GABAergic innervation plays a specific role in setting properties of synaptic transmission by comparing glutamatergic neurons, which were (1) alone (i.e., autaptic) on an astrocyte island, (2) in a pair with another glutamatergic neuron, or (3) in a pair with a GABAergic neuron. All three configurations formed spontaneously when hippocampal neurons were seeded at low density on astrocyte islands and could therefore be studied within the same neuronal cultures. Strikingly, we find that when comparing hippocampal neurons to autaptic neurons the frequency of mEPSCs was reduced in glutamatergic/GABAergic pairs, but not in homotypic glutamatergic pairs, and this decrease depended on $\mathrm{GABA}_{\mathrm{A}}$ receptor signaling, but not on electrical activity, indicating a role for spontaneous GABA release in downregulating mEPSC rates. Furthermore, only in such reciprocally connected glutamatergic/GABAergic pairs did deletion of synaptotagmin-1 lead to unclamping of mEPSCs, indicating that GABAergic innervation affects the operation of the glutamatergic release machinery.

\section{Materials and Methods}

Hippocampal cultures. For electrophysiology, isolated hippocampal neurons were plated on astrocyte microislands (Bekkers and Stevens, 1991). In short, hippocampi were dissected from postnatal day 1 (P1) NMRI pups or embryonic day 18 (E18) synaptotagmin-1-null mutant $(-/-)$ mice and control littermates $(+/+)$ of either sex, and collected in HBSS (Sigma), buffered with $7 \mathrm{~mm}$ HEPES. After removal of the meninges, hippocampi were minced and incubated for $20 \mathrm{~min}$ in $0.25 \%$ trypsin in HBSS at $37^{\circ} \mathrm{C}$. After washing, the neurons were triturated with firepolished Pasteur pipettes, counted, and plated in Neurobasal (E18 neurons) or Neurobasal-A (P1 neurons) medium (Invitrogen) supplemented with 2\% B-27 (Invitrogen), 1.8\% HEPES, 1\% glutamax (Invitrogen), $1 \%$ Pen/Strep (Invitrogen), and $0.2 \% \quad \beta$-mercaptoethanol. Neurons were plated at $2500 / \mathrm{cm}^{2}$ on microislands of mouse glia. Glial islands were obtained by first coating glass coverslips with $0.15 \%$ agarose. After drying and UV sterilization, custom-made rubber stamps were used to print dots (islands; diameter, 200-250 $\mu \mathrm{m}$ ) using a substrate mixture containing $0.25 \mathrm{mg} / \mathrm{ml}$ rat tail collagen and $0.4 \mathrm{mg} / \mathrm{ml}$ poly-Dlysine dissolved in $17 \mathrm{~mm}$ acetic acid; glial cells were plated at $4800 / \mathrm{cm}^{2}$. For active synapse quantification experiments, Synaptophysin-pHluorin (SypHy) cDNAs was subcloned into a pLenti vector, and viral particles were produced as described previously (Naldini et al., 1996). Neurons were transduced at $1 \mathrm{~d}$ in vitro (DIV1).

Electrophysiological recordings. Isolated and pairs of hippocampal neurons from postnatal NMRI pups, synaptotagmin-1-null mutant embryos and their wild-type (WT) littermates were recorded on DIV12-15. The patch-pipette solution contained the following (in $\mathrm{mM}$ ): $136 \mathrm{KCl}, 18$ HEPES, $4 \mathrm{Na}$-ATP, $4.6 \mathrm{MgCl}_{2}, 4 \mathrm{~K}_{2}$-ATP, 15 creatine phosphate, 1 EGTA, and $50 \mathrm{U} / \mathrm{ml}$ phospocreatine kinase (300 mOsm), pH 7.30. The external medium used contained the following components (in $\mathrm{mM}$ ): 140 $\mathrm{NaCl}, 2.4 \mathrm{KCl}, 4 \mathrm{CaCl}_{2}, 4 \mathrm{MgCl}_{2}, 10$ HEPES, 10 glucose (300 mOsm), pH 7.30. Cells were whole-cell voltage clamped at $-70 \mathrm{mV}$ with a double EPC-10 amplifier (HEKA Elektronik) under control of Patchmaster version $2 \times 32$ software (HEKA Elektronik). Currents were low-pass filtered at $3 \mathrm{kHz}$ and sampled at $20 \mathrm{kHz}$. Pipette resistance ranged from 3 to 5 $\mathrm{M} \Omega$. The series resistance was compensated to $85 \%$. Only cells with series resistances below $15 \mathrm{M} \Omega$ were included for analysis. All recordings were made at room temperature. Brief depolarization steps of the cell soma (from -70 to $0 \mathrm{mV}$ for $2 \mathrm{~ms}$ ) were used to initiate action potentialdependent (evoked) GABA and glutamate release [resulting in the measurements of evoked IPSCs (eIPSCs) and evoked EPSCs (eEPSCs), respectively]. The identity of recorded neurons could be confidently established by comparing decay time kinetics of recorded evoked and spontaneous release (see Results). Fitmaster version $2 \times 43$ was used for off-line analysis of evoked recordings. Spontaneous events were detected using the Mini Analysis program (Synaptosoft).

Islands containing one or two neurons were initially identified by visual inspection. Following successful whole-cell patch-clamp, the presence of visually undetected, and thus unclamped, neurons were ruled out based on the absence of multicomponent postsynaptic currents upon stimulation of action potentials in the patched cells. Multicomponent postsynaptic currents typically result from the excitation of an unclamped neuron, leading to a recurrent postsynaptic current in the patched cell. This criterion can thus be used to test for undetected neurons, provided that one of the patched neurons is glutamatergic and the synaptic strength between the interconnected neurons is sufficient to elicit action potentials. The latter can be assumed due to the indiscriminate formation of synapses between neurons on islands (see Results). In the case of isolated autaptic GABAergic neurons or GABAergic pairs, the absence of mEPSC events was used to rule out the presence of an undetected glutamatergic neuron.

Synapse counting. Neurons used for imaging were transduced with SypHy (Granseth et al., 2006) expressing lentiviral particles on DIV1. Isolated and pairs of hippocampal neurons from postnatal NMRI pups were recorded on DIV8-9. Either MPTS (8-methoxypyrene-1,3,6trisulfonic acid; $10 \mu \mathrm{M})$ or Alexa Fluor-568 $(5 \mu \mathrm{M})$ was added to the patch-pipette solution (see above) and infused into the neurons during whole-cell patch-clamp recordings. Spontaneous release was measured from both neurons, and they were stimulated to record evoked currents and identify their identity as glutamtergic or GABAergic. Train stimulation (300 action potentials (AP) at $40 \mathrm{~Hz}$ ) of either neuron was combined with imaging of SypHy to identify the population of active glutamatergic or GABAergic synapses. Imaging was performed using a dual-band filter (DA/FI-A-000, Semrock; excitation, $490 \mathrm{~nm}$ ). After the recording, the whole-cell patch pipettes were carefully retracted, thus exposing the total dendritic arborization, which could now be visualized using excitation of either MPTS $(395 \mathrm{~nm})$ or Alexa Fluor-568 $(560 \mathrm{~nm})$ residing inside the neurons (DA/FI/TR-A-000 triple-band filter, Semrock). The total number of synaptic connections was identified using superfusion of $\mathrm{NH}_{4} \mathrm{Cl}$, equilibrating the $\mathrm{pH}$ across all membranes to 7.4, thereby unquenching all SypHy-positive synaptic contacts. The $\mathrm{NH}_{4} \mathrm{Cl}$ medium used contained the following components (in $\mathrm{mm}$ ): $90 \mathrm{NaCl}, 50 \mathrm{~mm} \mathrm{NH}_{4} \mathrm{Cl}, 24$ $\mathrm{KCl}, 4 \mathrm{CaCl}_{2}, 4 \mathrm{MgCl}_{2}, 10$ HEPES, and 10 glucose, $\mathrm{pH}$ 7.20. Background correction was used to identify specific SypHy fluorescence by acquiring and subtracting a SypHy image before $\mathrm{NH}_{4} \mathrm{Cl}$ application, or train stimulation, with the same exposure time. Synapses were detected using automated synapse recognition software (Schmitz et al., 2011). First, the dendritic arborization of an isolated neuron or of each neuron within a pair, as revealed by MPTS or Alexa Fluor-568 imaging, was manually traced. SypHy fluorescent spots were automatically detected and included as representing synapses if they fulfilled the following three criteria: first, the $\mathrm{NH}_{4} \mathrm{Cl}$-induced increase in fluorescence intensity should be at least two times the SD above noise level. Second, the spot size should be at least $0.6 \mu \mathrm{m}^{2}$ to exclude spots representing smaller intracellular compartments or noise. Third, only spots appearing on the dendritic mask were included. The latter criterion prevents including SypHy fluorescent spots that are not colocalized with neuronal extensions (e.g., from expression in glial cells forming the microdot island). Synapses contacting each individual neuron were counted, and the total synapse number in pairs was found by summing the synapse number of both neurons. A small fraction $(\sim 10 \%$ of total) of synaptic contacts overlapped significantly with both dendritic masks. Experiments were conducted at room temperature on an inverted Zeiss Axiovert 200 microscope equipped with an LD LCI Plan-Apo $25 \times / 0.8$ numerical aperture oil-immersion objective (Carl Zeiss Microscopy). Fluorophores were excited by a monochromator (Polychrome V, TILL Photonics) controlled by TILLVision, and images $(1376 \times 1040$ pixels $)$ were acquired with a cooled digital 12-bit CCD camera (SensiCam, PCO-Tech).

Statistics. The results are shown as the average \pm SEM, with $n$ referring to the number of recorded isolated or paired neurons for each group, unless otherwise indicated. For analysis, datasets were first tested for normal distribution. If data were normally distributed (or attained normal distributions using log transformation; normality was tested with an 
equal variance test), we used one-way ANOVA to test differences between the different groups. If a statistical difference was found within the population of groups using ANOVA, we tested the difference between individual group means using Tukey's test. Datasets that did not show normal distribution were tested using the Kruskal-Wallis test. If a statistical difference was found within the population of these groups, we tested the differences between individual group medians using Dunn's method. Two-tailed $t$ tests were used to compare two groups. Significance was assumed when $p<$ 0.05 . Statistical testing was performed using SigmaPlot 11 (SYSTAT). In figures, the significance levels are indicated by asterisks, as follows: ${ }^{\star} p<0.05 ;{ }^{\star *} p<0.01$; and ${ }^{\star * *} p<0.001$.

\section{Results}

Functional autaptic and interneuronal synaptic connections form without preference within reciprocally connected neuronal pairs

To study the interdependence of glutamatergic and GABAergic synaptic transmission in minimal neuronal networks, we here compared microdot glial islands containing isolated (autaptic) glutamatergic or GABAergic neurons from NMRI mice (Fig. $1 A, C$ ) with glial islands containing two reciprocally connected neurons (Fig. 1B). The minimal networks formed by two neurons were either homotypic pairs (i.e., two glutamatergic or two GABAergic neurons) or "mixed pairs" containing one glutamatergic and one GABAergic neuron (Fig. 1C). All configurations formed spontaneously when hippocampal neurons were seeded at low density on astrocyte islands, and they could therefore be studied and compared within the same neuronal

cultures. The identity of cultured hippocampal neurons (either GABAergic or glutamatergic) is difficult to recognize based on morphology only; therefore, their classification could only be determined with certainty during whole-cell recordings. To assess synaptic connectivity within a minimal network, both cells were measured simultaneously using whole-cell patch-clamp of the cell soma, and subsequent brief depolarizations were used to elicit an action potential in either of the connected neurons (Fig. $1 D)$. Since we used high internal chloride concentration during whole-cell recordings, both glutamatergic and GABAergic release events were detected as inward currents. However, the identity of recorded neurons could be easily established by comparing decay time kinetics of recorded evoked and spontaneous release events; GABAergic postsynaptic currents decay much slower (Fig. $1 H$ ) compared with glutamatergic postsynaptic currents (Figs. $1 G$, $2 B, C$, spontaneous events; Wilcox et al., 1994; Toonen et al., 2006). Acute block of GABAergic and glutamatergic neurotransmission by bicuculline and CNQX in mixed pairs confirmed accurate identification of glutamatergic and GABAergic neurons based on those criteria (Fig. $2 F$ ). Furthermore, in each recording the absence of multicomponent postsynaptic responses after stimulation was used to rule out the presence of an undetected, solated neurons

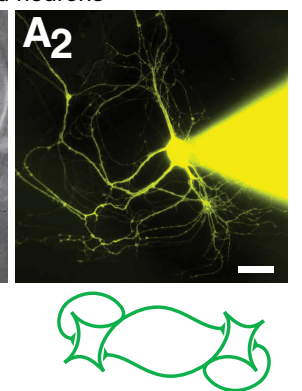

Glutamatergic

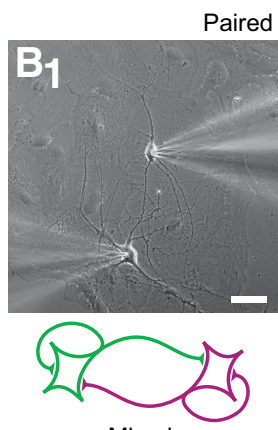

Mixed
Paired neurons

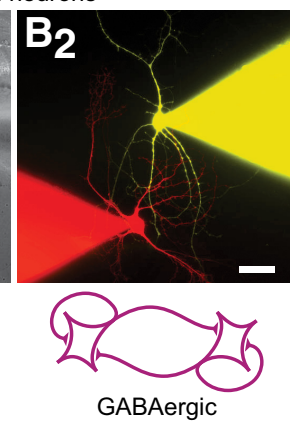

GABAergic

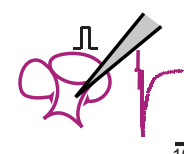

GABAergic
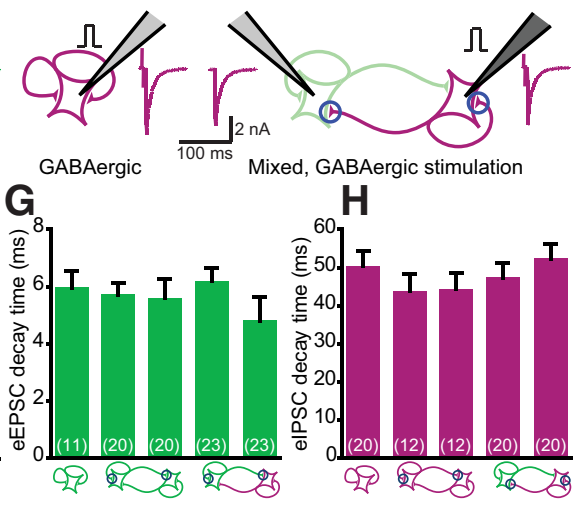

ixed, GABAergic stimulation

H
F

Figure 1. Reciprocal connectivity within minimal neuronal networks. $\boldsymbol{A}, \boldsymbol{B}$, Bright-field and fluorescence images of an isolated $(\boldsymbol{A})$ and a pair consisting of two connected neurons (B, DIV14). Alexa Fluor-467 (red) or lucifer yellow (yellow) was used to ( evoked charge was comparable between the different groups (glutamatergic evoked charge: isolated, $0.042 \pm 0.0085$ pC; homo-

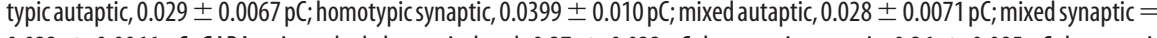
作 $0.0066 \mathrm{pC}$; GABAergic evoked charge: isolated, $0.27 \pm 0.032 \mathrm{pC}$; homotypic autaptic, $0.36 \pm 0.085 \mathrm{pC}$; homotypic synaptic, $0.26 \pm 0.089$ pC; mixed autaptic, $0.28 \pm 0.049 \mathrm{pC}$; mixed synaptic, $0.38 \pm 0.058 \mathrm{pC}$ ). Differences in amplitude and decay time of eEPSCs and eIPSCs between different groups were tested using one-way ANOVA ( $p>0.05)$.

unclamped (third) neuron, so that only pairs of neurons were studied (see also Materials and Methods).

Stimulating isolated glutamatergic or GABAergic neurons during whole-cell patch-clamp resulted in autaptic eEPSCs (Fig. $1 D$, isolated green neuron) or eIPSCs (Fig. $1 D$, isolated magenta neuron), respectively. Excitation of either neuron within a pair resulted in autaptic and interneuronal synaptic evoked responses in the stimulated and unstimulated neuron, respectively (Fig. 1D, mixed pairs with stimulation of glutamatergic or GABAergic neuron shown separately; left and right pair). Glutamatergic neurons in either homotypic pairs (i.e., with another glutamatergic neuron) or mixed pairs (with a GABAergic neuron) showed comparable autaptic and synaptic eEPSC amplitudes, which were also indistinguishable from eEPSCs in purely autaptic neurons (Fig. $1 E$ ). The same was found for GABAergic neurons (Fig. $1 F$ ), indicating that both neuronal types form functional autaptic and synaptic connections without preference. Furthermore, no differences were found in the decay time kinetics of eEPSCs and eIPSCs, suggesting postsynaptic receptor composition and asynchronous release were comparable between all groups (Fig. $1 G, H)$. These data deviate somewhat from previous reports from the Chapman laboratory, where interneuronal synaptic EPSCs were reported to be on average smaller than autaptic EPSCs 


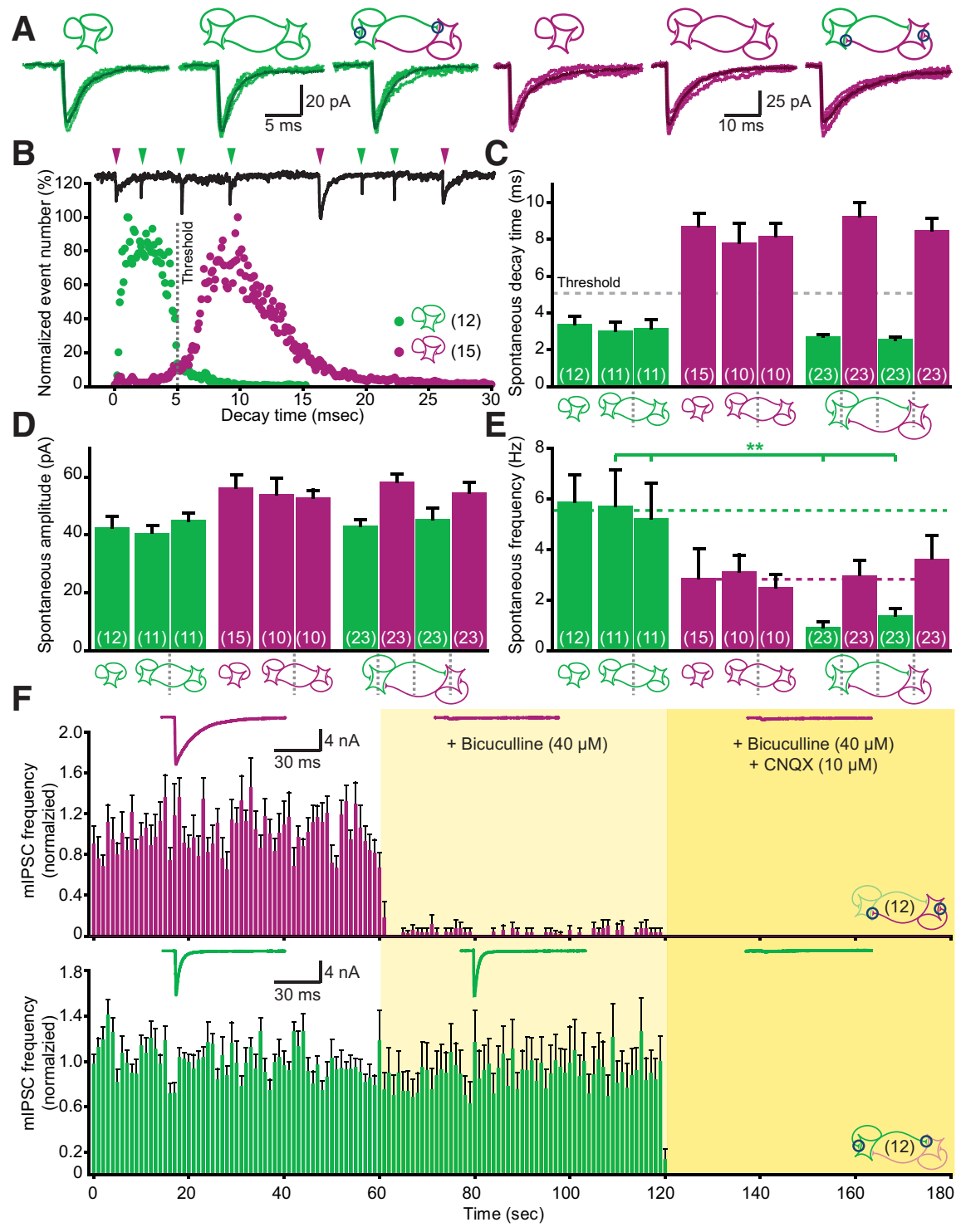

Figure 2. GABAergic input attenuates mEPSC frequency in mixed pairs. $A$, Example traces of spontaneous glutamatergic ( $\mathrm{mEPSC}$, green) and GABAergic (mIPSCs, magenta) release events in all experimental groups. Thick traces represent the averaged spontaneous release event in each condition. Internal recording solution contained high $\left[\mathrm{Cl}^{-}\right]$, thus both $\mathrm{mEPSCS}$ and mIPSCs were detected as inward currents. $B$, A threshold of $5 \mathrm{~ms}$ was used to segregate mEPSCs and mIPSCs in mixed pairs (histogram, bin size $=0.1 \mathrm{~ms}$ ). Inset, $\mathrm{mEPSC}$ (green arrowheads) and mIPSC (magenta arrowheads) events in mixed pairs. C, No network-induced changes were found in spontaneous decay time kinetics using threshold segregation between mEPSCs and mIPSCs in mixed pairs. $\boldsymbol{D}$, Network association did not affect mIPSC or mEPSC amplitude. $\boldsymbol{E}$, Heterosynaptic connectivity in mixed pairs attenuates mEPSC release frequency $(p<0.01$, Student's $t$ test comparing average mEPSC frequency, averaged from both cells within a pair, in homotypic pairs with average mEPSC frequency in mixed pairs). $\boldsymbol{F}$, Acute application of bicuculline ( $40 \mu \mathrm{m}$ ) blocked both mIPSCs and elPSCs in mixed pairs (magenta), but mEPSCs and eEPSCs release was unaffected (green). Consecutive application of bicuculline (40 $\mu \mathrm{M})$ and $\mathrm{CNQX}(10 \mu \mathrm{M})$ blocked all spontaneous release. Insets show elPSCs and eEPSCs before and during bicuculline or bicuculline/CNQX application. Note that bicuculline only blocks mIPSCs, indicating that the segregation based on decay time accurately distinguishes between mEPSCs and mIPSCs. The remaining events during bicuculline represent mis-sorted mEPSCs, which are blocked during bicuculline/CNQX application.

within neuronal pairs (Liu et al., 2009, 2013). The reason for this difference is unknown, but it probably results from differences in the protocols used. For instance, the previous reports made use of poly-lysine spraying, which creates astrocyte islands of varying size (100-1000 $\mu \mathrm{m}$; Liu et al., 2013), whereas we used poly-lysine stamping, which results in islands of more constant size (200$250 \mu \mathrm{m})$. Since it was hypothesized that the larger autaptic EPSCs are caused by proximity, not by a preference for the autaptic connection type (Liu et al., 2013), the use of larger islands might favor autaptic over synaptic EPSCs. Whatever the reason for this difference, in our experiments two neurons forming a pair were as strongly coupled to the other neuron as they were to themselves.

\section{GABAergic input attenuates mEPSC frequency in mixed pairs}

To study the effect-if any- of the reciprocally connected neuron within a minimal network on spontaneous release, we recorded from unstimulated isolated and reciprocally connected neurons. Spontaneously occurring miniature GABAergic (mIPSCs) and glutamatergic (mEPSC) release events recorded from isolated (autaptic) neurons could be clearly separated based on their decay time kinetics (Fig. $2 A, B)$. In mixed pairs, where mIPSCs and mEPSCs were measured simultaneously, we therefore used a threshold of $5 \mathrm{~ms}$ to segregate mEPSCs and mIPSCs (tolerating a mis-sorting error of $6.5 \%$ for mEPSCs and 3.5\% for mIPSCs). Decay time and amplitude of mEPSCs were comparable between all groups (Fig. $2 C, D$ ), suggesting that the presence or identity of the reciprocally connected neuron did not lead to major postsynaptic changes. The same was found for GABAergic neurons (Fig. $2 C, D)$. No difference was found in spontaneous release frequency between isolated and homotypic pairs of glutamatergic neurons (Fig. 2E). However, a pronounced decrease in both autaptic and interneuronal synaptic mEPSC frequency was found in mixed (glutamate/GABA) pairs, indicating that the presence of a reciprocally connected GABAergic neuron resulted in the attenuation of spontaneous release events from the excitatory neuron (Fig. 2E, right). This contrasts with the finding that the evoked EPSCs had similar amplitudes in mixed pairs, autaptic neurons, and homotypic glutamatergic pairs (Fig. 1E), indicating that, specifically, the spontaneous glutamate release was changed in mixed pairs. In fact, mIPSC and mEPSC frequencies were inversely correlated within mixed pairs (NMRI mice: Spearman's rank correlation, $r=-0.455, p=$ 0.0001; C57BL/6 mice: Spearman's correlation coefficient $=-0.405, p=$ 0.001 ; see Fig. $5 C, D$ ), indicating that the extent to which mEPSC frequency is downregulated depends on the strength of spontaneous GABAergic neurotransmission, which varies between mixed pairs. In contrast, mIPSC frequency was unchanged among autaptic GABAergic neurons, GABAergic homotypic pairs, and mixed pairs (Fig. $2 E$ ), showing that the regulation is only on the glutamatergic, not on the GABAergic, neuron within mixed pairs. 
To determine whether the attenuation of mEPSC release is due to ongoing, current GABAergic inhibition, we used acute application of the competitive $\mathrm{GABA}_{\mathrm{A}}$ receptor blocker bicuculline while recording mixed pairs. Acute bicuculline application blocked both evoked and spontaneous IPSCs, but did not affect mEPSC frequency (Fig. $2 F$, insets are evoked currents), establishing the validity of our identification of mEPSCs and mIPSCs, and ruling out an acute role of $\mathrm{GABA}_{\mathrm{A}}$ receptor signaling in negatively regulating mEPSC release in mixed pairs. Successive application of bicuculline and CNQX blocked all spontaneous events, indicating that the recorded mEPSCs are AMPA/kainate receptor-dependent release events. Note that the remaining mIPSCs during bicuculline application represent mis-sorted mEPSCs, which are blocked during bicuculline/CNQX application (Fig. 2F).

\section{Long-term spontaneous GABAergic} inhibition is necessary and sufficient to control spontaneous glutamatergic release in mixed pairs

Since attenuation of acute GABAergic signaling does not affect mEPSC release in mixed pairs (Fig. 2F), we investigated whether the reduction of mEPSC frequency in mixed pairs depends on longterm $\mathrm{GABA}_{\mathrm{A}}$ receptor signaling. To test this, we again used bicuculline, but now studied the effects of prolonged inhibition of GABAergic signaling on mEPSC release in established pairs. Bicuculline was added to the cultures at DIV10-11 (i.e., when synapses had already been established), and after $3 \mathrm{~d}$ paired neurons were recorded in normal external solution (without bicuculline). Bicuculline stayed active in the culture medium during the $3 \mathrm{~d}$ of incubation, since local application of this medium completely blocked eIPSC amplitude (Fig. $3 A$ ). Inhibition of $\mathrm{GABA}_{\mathrm{A}}$ receptor signaling for $3 \mathrm{~d}$ completely reversed the inhibition of mEPSC frequency in mixed pairs (Fig. $3 B$; average mEPSC in mixed pairs pretreated with bicuculline compared with untreated pairs: $p<0.001, t$ test; see Fig. $6 A 1$ for similar effect in C57BL/6 mixed pairs).

Since bicuculline blocks $\mathrm{GABA}_{\mathrm{A}}$ receptors, either evoked or spontaneous GABA release might regulate mEPSC release. To segregate between these two possibilities, we used long-term tetrodotoxin (TTX) application to block action potentialdependent release. Also, TTX was still active after $3 \mathrm{~d}$ of incubation, since local application of TTX-containing medium blocked both action potential-dependent eEPSCs and eIPSCs (Fig. 3C). However, long-term TTX treatment did not reverse the inhibition of mEPSC frequency in mixed pairs (Fig. 3D). Therefore, spontaneous GABA release acts to confine mEPSC release rates. It should be borne in mind that TTX also blocks action potentials in the glutamatergic neuron, which might affect mEPSC frequencies in older cultures, but in that case an increase should be seen (Wierenga et al., 2006), so this is unlikely to con- found our conclusion. This experiment further rules out that the effect of bicuculline could be mediated by a changed firing pattern in the glutamatergic neuron (Fig. $3 E$ ).

Strikingly, the presence of a GABAergic neuron affected mEPSC release rates equally in autaptic and interneuronal synaptic connections (Figs. 2E, 3B). This shows that the changes to the glutamatergic neuron are global and not caused by any inherent differences between autaptic and interneuronal synaptic connections. This finding further rules out the scenario of a very local retrograde inhibitor from the GABAergic neuron affecting mEPSC frequencies in a synapse-specific manner.

In sum, we find that, in mixed pairs, the GABAergic neuron reduces the mEPSC rate in both autaptic and interneuronal synaptic connections in the reciprocally connected glutamatergic neuron via a mechanism that requires long-term $(3 \mathrm{~d}) \mathrm{GABA}_{\mathrm{A}}$ activation. This result-and the negative correlation between mIPSC and mEPSC frequencies — indicates that miniature events are interdependent within neuronal networks.

\section{Spontaneous release rates are not proportional to the number of active synapses}

A reduced mEPSC frequency in mixed pairs compared with a homotypic glutamatergic pair might be expected based on the fact that, as a first approximation, half of the incoming connections into any of the neurons are expected to be glutamatergic, 
whereas the rest are GABAergic. Yet, the mIPSC frequency was unchanged in mixed pairs compared with either homotypic GABAergic pairs or autaptic neurons, which, together with the effect of long-term bicuculline application, indicates that a specific process acts to control mEPSC frequencies in mixed pairs. To scrutinize the relationship between miniature rate and synapse number, we combined patch-clamp with quantification of the synapse population.

We quantified connections in autaptic neurons and in neuronal pairs using expression of the $\mathrm{pH}$-sensitive fluorescent marker SypHy. In this construct, the $\mathrm{pH}$-sensitive variant of GFP (pHluorin) is coupled to the luminal domain of Synaptophysin (Granseth et al., 2006), allowing us to identify synaptic connections by imaging. Following patch-clamp, we measured spontaneous events from both neurons and then stimulated either neuron to identify their identity as glutamatergic or GABAergic. To identify synapses formed by each neuron, we stimulated the two neurons sequentially by trains of action potentials (300 AP at $40 \mathrm{~Hz}$ ) while imaging. Afterward, an ammonium solution at $\mathrm{pH}$ 7.2 was superfused to identify the total synapse number. Finally, we withdrew both pipettes and identified the branch pattern of each neuron by imaging two different fluid phase markers that were infused into the neurons during patch-clamp recording. This pattern was used to create a dendritic mask, which was used to ensure that identified spots were bona fide synapses and not SypHy expressed in the underlying glial cells (see Materials and Methods). Synapses were identified as distinct spots in background corrected images (Fig. 4A2,B2; images in the presence of ammonium were subtracted by prestimulation images; similarly, images obtained before the $300 \mathrm{AP}$ train were subtracted from images obtained during the train) using published software (Schmitz et al., 2011). Figure 4, A3 and B3, shows the mask and identified synapses in an autaptic neuron and a pair, respectively, following ammonium superfusion. Figure 4, A4 and B4, shows the mask and synapses obtained by train stimulation of one neuron in the same pair (indicated by asterisk).

In neuronal pairs - whether homotypic, glutamatergic, or mixed - we found that the total number of synapses (identified by ammonium) was approximately doubled compared with autaptic neurons (Fig. 4C). When evaluating these data, the detection limit of pHluorin imaging should always be kept in mind, as well as the fact that some synapses might be postsynaptically (Shen et al., 2006) or presynaptically (Crawford et al., 2012) silent. Our numbers overall agree with—and are even a bit higher than-previously reported data using immunostaining against VGLUT (Liu et al., 2009, 2013), indicating sufficient detection.

To compare the number of glutamatergic synapses to the spontaneous release frequencies, we summed the total number of glutamatergic synapses on an island (i.e., from both neurons, in case of a glutamatergic pair) using the synapse numbers identified during the $40 \mathrm{~Hz}$ train (since those synapses could be unequivocally identified as glutamatergic, based on the identity of the patched cell). Likewise, we summed up the mEPSC frequencies from both neurons, in case of a pair, and plotted the summed mEPSC frequencies as a function of the number of glutamatergic synapses (Fig. 4F). The same was done for mIPSCs and GABAergic synapses (Fig. 4G). When connecting the points representing autaptic neurons with those representing homotypic pairs, it can be appreciated that miniature frequencies tended to saturate (Fig. 4F, G). This effect was stronger for GABAergic neurons. The point from the mixed pairs falls nicely on the same curve as far as the mIPSC frequency is concerned (Fig. 4G), whereas it falls below the curve in the case of mEPSCs (Fig. 4F). Thus, the reduction of mEPSC frequency in mixed pairs is more severe than expected due to the decrease in the number of synapses.

The neurons used for imaging were younger (DIV8 -9) than those used previously for purely electrophysiological measurements (DIV12-15), because the branch pattern of older neurons was difficult to identify. To investigate the relationship between spontaneous and evoked release in the older neurons, we summed up all evoked currents (trans-synaptic and autaptic, from both neurons in the case of pairs) on an island and plotted the sum of spontaneous frequencies against the sum of evoked currents for the data presented in Figure 2. This resulted in very similar plots (Fig. $4 H, I$ ), but with overall higher spontaneous release rates because of the age of the neurons. Also in this set of data, the mEPSC frequency in mixed pairs fell below the curve between the autaptic and glutamatergic pair (Fig. 4H), whereas the mIPSC frequency in mixed pairs was above the curve (Fig. $4 I$ ). This plot makes a similar point as the synapse-counting experiment and indicates that the mEPSC depression is stronger in the older neurons. Note that if the mEPSC frequency from the mixed pairs was recalculated according to the effect of bicuculline (Fig. 3), the mEPSC release frequency in mixed pairs would be close to the curve connecting autaptic neurons and homotypic glutamatergic pairs (Fig. $4 \mathrm{H}$, gray arrow and square).

Overall, the lower mEPSC frequency in mixed pairs cannot be explained only by a lower number of glutamatergic synapses in mixed neuronal pairs compared with glutamatergic pairs, indicating a specific action of GABA on glutamatergic miniature release probability.

\section{Enhanced spontaneous release typical for synaptotagmin-1- null mutants depends on mixed connectivity}

Several reports found that elimination of synaptotagmin-1 caused disinhibition of spontaneous release in neuronal networks, but not in isolated neurons (for references, see Introduction). Here, we scrutinized the minimal requirements to unveil the characteristic disinhibition of spontaneous release in the synaptotagmin-1-null mutant. Again, we found a strong reduction in $\mathrm{mEPSC}$ frequency in mixed pairs from control wild-type littermates $\left(\right.$ Syt- ${ }^{+/+} ; \mathrm{C} 57 \mathrm{BL} / 6$ mouse strain; Fig. 5B1), which is in agreement with our previous observations in NMRI mice (Fig. $2 E$ ). Upon deletion of synaptotagmin-1, no disinhibition of mEPSC frequencies was observed in isolated synaptotagmin-1null mutant $\left(S y t-1^{-1-}\right)$ glutamatergic neurons or in homotypic pairs (Fig. 5B1,B2, compare left and right panels; for example traces from homotypic pairs, see Fig. 5A1,A2, top traces), which is in line with previous reports. However, a striking increase in the frequency of both mIPSCs and mEPSCs was unveiled in mixed pairs (Fig. 5B1,B2, compare left and right mixed pair data; for example traces from $S y t-1^{-1-}$ mixed pairs, see Fig. $5 A 1, A 2$, bottom traces), showing that reciprocal connectivity between at least one GABAergic and one glutamatergic neuron is sufficient to instigate this Syt-1 ${ }^{-1-}$ phenotype in glutamatergic neurons. In addition, the inverse relationship between mIPSC and MEPSC frequencies found in wild-type mixed pairs from NMRI (Fig. 5C) and C57BL/6 (Fig. 5D) mice was lost in Syt- $1^{-1-}$ mixed pairs (Fig. 5E; Spearman's correlation coefficient $=0.228, p=0.145$ ), suggesting lack of GABAergic-induced control over mEPSC release.

Since mixed connectivity (1) induces disinhibition of spontaneous release in Syt-1 ${ }^{-1-}$ neurons (Fig. $5 A, B$ ) and (2) attenuates mEPSC release using $\mathrm{GABA}_{\mathrm{A}}$ receptor signaling (Fig. $3 B$ ), the question arises of whether a common mechanism is involved. We hypothesized that after bicuculline treatment of $S y t-1^{-1-}$ mixed pairs, 

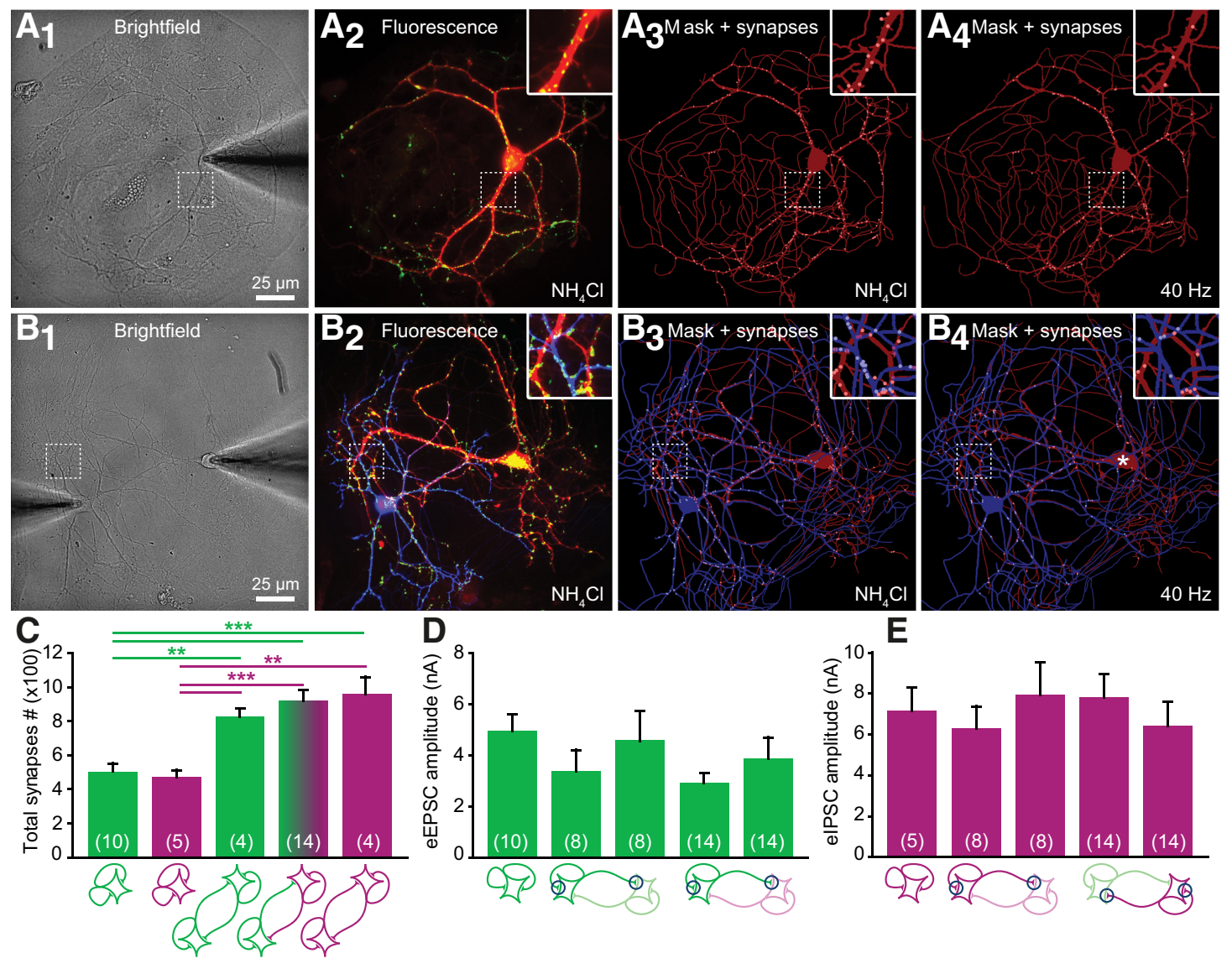

E
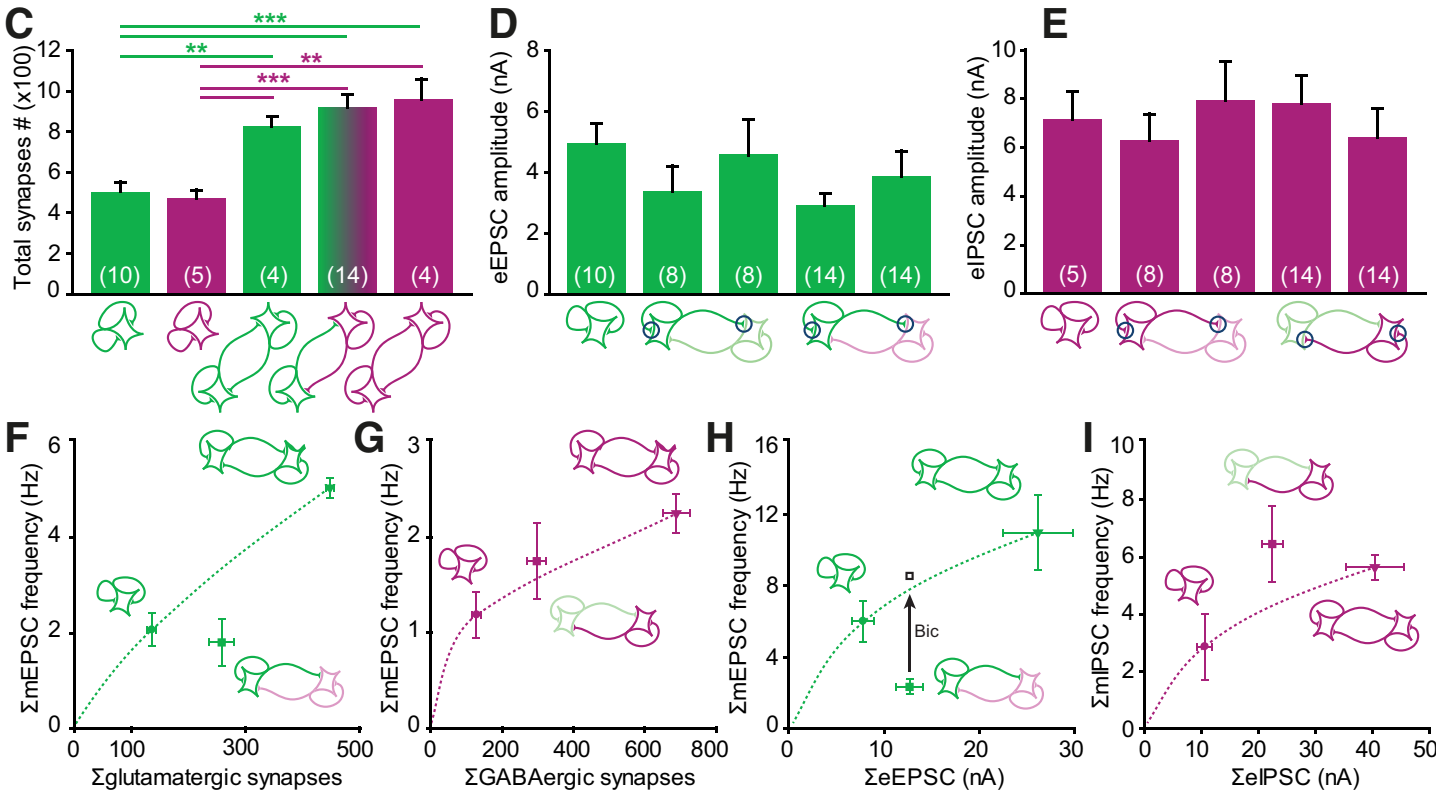

Figure 4. Counting synapses in isolated (autaptic) neurons and interconnected neuronal pairs. $\boldsymbol{A}, \boldsymbol{B}$, The total number of synaptic connections formed by isolated ( $\boldsymbol{A}$ 1) or pairs ( $\boldsymbol{B}$ 1) of neurons was determined using superfusion of $\mathrm{NH}_{4} \mathrm{Cl}$, herewith unquenching all SypHy-positive synaptic contacts (DIV8; $A 2, B 2$ ). MPTS (red) or Alexa Fluor-568 (blue) was infused during whole-cell recordings to visualize dendritic arborization. Recording electrodes were retracted before imaging, exposing the complete arborization. $\mathbf{A}, \mathbf{B} 3$, Manual tracking of the neuronal arborization resulted in dendritic masks of the isolated autaptic neurons and of neuronal pairs. Filled circles represent SypHy-positive puncta that were automatically identified and colocalized with the dendritic mask (Schmitz et al., 2011). A3, SypHy-positive puncta representing autaptic connections (orange dots) based on colocalization with a red dendritic mask of an isolated autaptic neuron. B3, SypHypositive spots in a pair, representing either autapses or interneuronal synapses on the red neuron (orange dots) or the blue neuron (light blue dots). A small fraction of synapses were allocated to both neurons due to dendritic overlap (purple dots). A4, B4, High-frequency trains ( $40 \mathrm{~Hz}, 300$ stimulations) were used to determine the number of active GABAergic or glutamatergic synapses. C, The total number of SypHy-positive spots that appear during superfusion with $\mathrm{NH}_{4} \mathrm{Cl}$ is comparable between glutamatergic and GABAergic isolated neurons. Pairs of neurons establish twice as many synaptic connections, suggesting the number of synaptic connections made per neuron is constant (0ne-way ANOVA $(p<0.05)$, followed by Tukey's test for subsequent pairwise comparisons). $\boldsymbol{D}$, Evoked amplitude is comparable between autaptic and synaptic connections in glutamatergic neurons in isolation and minimal networks (DIV8 -9), indicating no strong preference in formation of functional autaptic or synaptic glutamatergic connections (Kruskal-Wallis test, $p>0.05$ ). $\boldsymbol{E}$, Similarly, evoked amplitude is comparable between autaptic and synaptic connections in GABAergic neurons in isolation and minimal networks (Kruskal-Wallis test, $p>0.05$ ). $\boldsymbol{F}, \mathbf{G}$, Correlation plot between the total number of active synapses (assessed using $40 \mathrm{~Hz}$ trains) and the total $\mathrm{mEPSC} / \mathrm{mIPSC}$ release frequency in all groups (frequencies summed up from both neurons in the case of a pair). $\boldsymbol{F}, \boldsymbol{G}$, The total mIPSC frequency and GABAergic synapse number correlate in mixed pairs, but the total mEPSC frequency in mixed pairs was lower than expected based on number of glutamatergic synapses. $\boldsymbol{H}, \boldsymbol{I}$, Correlation plot between the summed eEPSC/elPSC amplitudes and the total $\mathrm{mEPSC} / \mathrm{mIPSC}$ release frequency in all groups (frequencies summed up from both neurons in the case of a pair). The mEPSC frequency was much lower than expected based on the summed eEPSC amplitude in mixed pairs, while the mIPSC frequency was more consistent with the eIPSC amplitude found in mixed pairs. Long-term treatment with bicuculline blocks the GABAergic inhibition of mEPSC frequency (Fig. 3B), herewith shifting the mEPSC frequency toward the predicted value ( $\boldsymbol{H}$, gray arrow marked 'Bic' and square).

a shared underlying mechanism would exclude further enhancement of mEPSC release. Long-term bicuculline treatment $(3 \mathrm{~d})$ confirmed the involvement of $\mathrm{GABA}_{\mathrm{A}}$ receptor signaling in regulating mEPSC release in mixed pairs from wild-type C57BL/6 littermates $\left(\right.$ Syt- $1^{+/+} ;$Fig. 6A1). Furthermore, long-term bicuculline treatment induced an additional increase of mEPSC release in Syt- $1^{-/-}$mixed pairs (Fig. $6 A 2$ ). Thus, $\mathrm{GABA}_{\mathrm{A}}$ receptor-dependent attenuation and the typical Syt-1 ${ }^{-1-}$ disinhibition apparently comprise two independent pathways affecting spontaneous glutamatergic release in mixed pairs (Fig. 6B). 

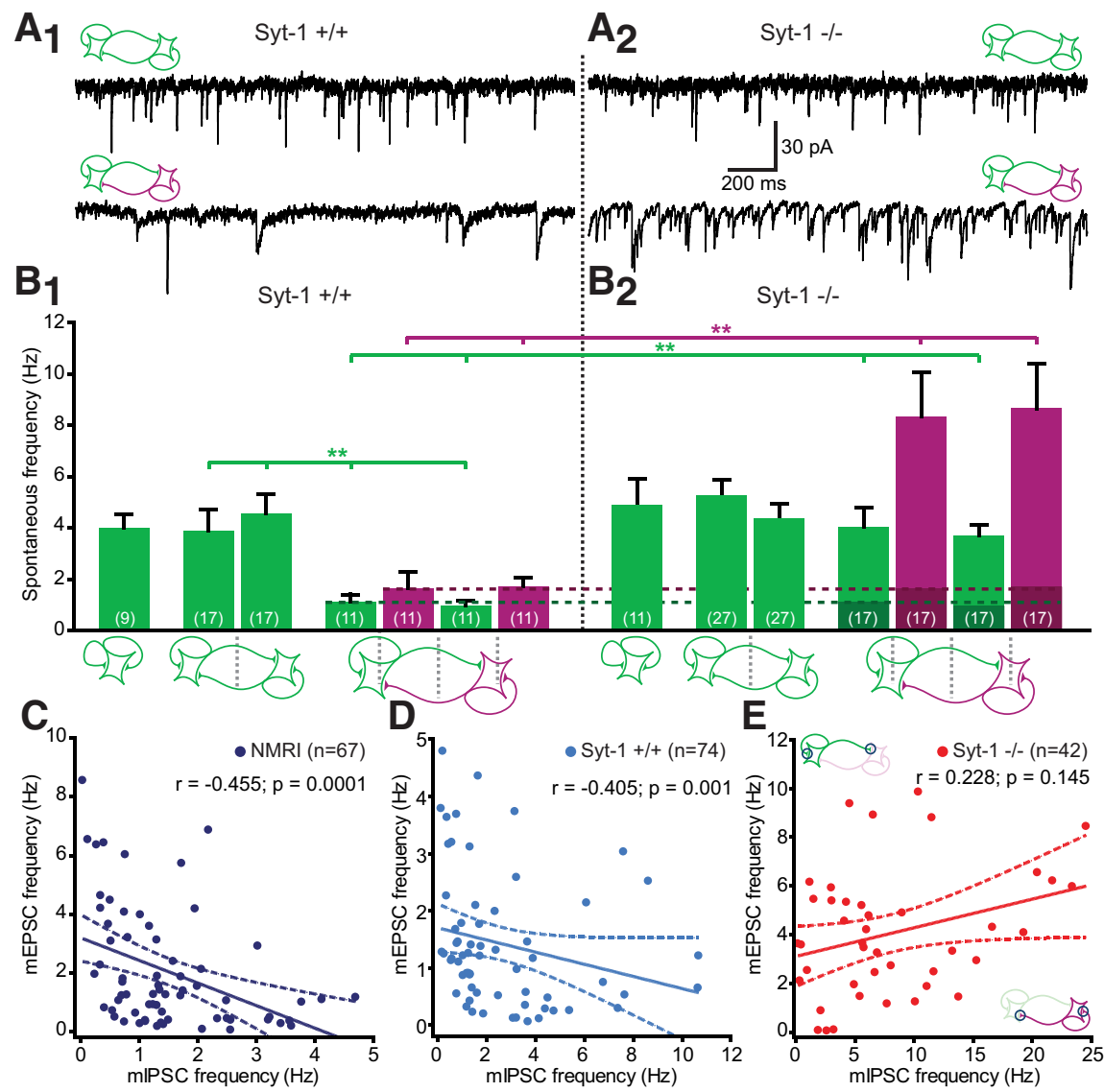

Figure 5. Enhanced spontaneous release in synaptotagmin-1-null mutants depends on 'mixed' connectivity. A1, Example traces of spontaneous release in wild-type homotypic (Syt- $1^{+/+}$, top trace) and mixed pairs (bottom trace). Note the discrete difference in kinetics between mEPSCs and mIPSCs in the mixed pair. A2, Example traces of spontaneous release in synaptotagmin1-null (Syt- $1^{-1-}$ ) mutant homotypic (top trace) and mixed pairs (bottom trace). B1, B2, No difference was found in mEPSC frequency between isolated and homotypic pairs from wild-type and synaptotagmin-1-null neurons. Note that the GABAergic inhibition of $\mathrm{mEPSC}$ frequency is also present in mixed pairs from wild-type $\left(S y t-1^{+/+}\right) \mathrm{C} 57 \mathrm{BL} / 6$ mice $(\boldsymbol{B} 1, p<0.01$, Student'st test comparing average mEPSC frequency in homotypic pairs with average $\mathrm{mEPSC}$ frequency in mixed pairs). $\boldsymbol{B 2}$, The increased spontaneous release typical for synaptotagmin-1-null mutants is exposed only in mixed pairs ( $p<0.01$; Student's t test comparing average $\mathrm{mEPSC}$ and $\mathrm{mIPSC}$ frequencies between control and Syt- $1^{-1-}$ mixed pairs). $C, D, \mathrm{mEPSC}$ and $\mathrm{mIPSC}$ frequencies were inversely correlated in mixed pairs from wild-type NMRI mice (C, Spearman's rank correlation $=-0.455, p=0.0001)$ and in mixed pairs form wild-type C57BL/6 mice ( $\boldsymbol{D}$, Spearman rank correlation $=-0.405, p=0.001)$. $\boldsymbol{E}$, Correlation between $\mathrm{mEPSC}$ and mIPSC frequencies was absent in synaptotagmin-1-null mutant mixed pairs (Spearman's rank correlation: $r=0.228, p=$ 0.145).

Spontaneous release depends on intracellular calcium levels, though with a lower apparent $\mathrm{Ca}^{2+}$ cooperativity compared with evoked synaptic release (Lou et al., 2005). Therefore, an increase in basal calcium concentration could explain the increased spontaneous release frequency found in $S y t-1^{-1-}$ mutant mixed pairs. To test the role of basal calcium in regulating spontaneous release in homotypic and mixed hippocampal pairs, we preincubated cells with BAPTA-AM (a high-affinity, membrane-permeant calcium chelator) and subsequently recorded spontaneous release. The absence of evoked release confirmed the efficiency of the treatment. The mEPSC frequency in homotypic glutamatergic pairs pretreated with BAPTA-AM was strongly decreased compared with untreated neurons (compare Figs. 6C1, $5 A 1, B 1, B 2)$, showing that a significant portion of spontaneous release events in homotypic pairs are calcium dependent. This decrease in mEPSC and mIPSC frequency upon BAPTA-AM treatment agrees with several previous reports (Xu et al., 2009; Groffen et al., 2010; Nosyreva and Kavalali, 2010; Yang et al., 2010; Williams et al., 2012), but appears to contradict the findings of Vyleta and Smith (2011), who reported no effect of BAPTA-AM on mEPSC frequency and concluded that mEPSCs are stimulated by activation of the calcium-sensing receptor. The reason for this discrepancy remains unknown, but it is possible that different culture or measurement conditions might result in different proportions of calcium-dependent and calcium-independent miniature events.

In mixed pairs, a decrease in both mEPSC and mIPSC release to similar frequencies was found. Since BAPTA-AM eliminated the difference in spontaneous release rate between homotypic glutamatergic and mixed pairs, a difference in calcium homeostasis might be involved in setting the different miniature rates. Importantly, the typical Syt-1 $1^{-1-}$ disinhibition of spontaneous release found in mixed pairs persisted after pretreatment with BAPTA-AM (Fig. $6 C 2$ ), indicating that this phenotype depends on an increased spontaneous release willingness of synaptic vesicles.

Overall, we show that a minimal neuronal network consisting of a pair of reciprocally connected glutamatergic/GABAergic neurons is sufficient to reveal the Syt-1 ${ }^{-1-}$ unclamping phenotype, which is absent both in glutamatergic pairs and glutamatergic autaptic neurons. This might be caused partly by the occlusion of the synaptotagmin-1 phenotype due to the higher overall mEPSC rate in glutamatergic pairs and glutamatergic autaptic neurons, partly by a different operation of the release machinery.

\section{Discussion}

We investigated the interdependence of spontaneously occurring glutamatergic and GABAergic release events in pairs of hippocampal neurons growing on astrocyte islands. We found a marked decrease in mEPSC frequency in mixed glutamatergic/GABAergic interconnected pairs growing on astrocyte islands, and a negative correlation between mEPSC and mIPSC frequency. Interdependence of mEPSC and mIPSC frequency might be expected when neurons grow in a limited environment, because of competition for space during synaptogenesis. However, the counting of synapses using synaptophysin-pHluorin-or comparing them to the evoked release amplitudeshowed that the mEPSC rate does not follow in a simple way from the number of active synapses in mixed neuronal pairs (Fig. $4 F, H)$. Whereas a somewhat lower mEPSC rate in mixed pairs compared with homotypic glutamatergic pairs was expected due the lower number of glutamatergic synapses, this could not explain the reduction when compared with autaptic glutamatergic neurons, or the fact that long-term (from DIV10) bicuculline application increased the mEPSC frequency without any significant changes to evoked release. Strikingly, long-term bicuculline treatment restored the mEPSC frequency to expected values when considering the evoked release amplitude (Fig. $4 H$ ). The 
specificity of the interdependence of mEPSC and mIPSC frequencies is indicated by the fact that the frequency of mIPSCs was unchanged, regardless of whether the GABAergic neuron was in an autaptic situation, in a homotypic (GABAergic) pair, or in a mixed pair. Moreover, the total mIPSC rate seemed to follow from the number of GABAergic synapses (Fig. 4G), indicating that whereas the GABAergic neuron affects spontaneous release from the glutamatergic neuron, the opposite is not true.

Thus, our results appear inconsistent with a scenario where the spontaneous release frequency follows from simple competition between the formation of GABAergic and glutamatergic synapses, even though it also does not rule out that such a competition could exist. Our findings point to a specific downregulation of mEPSC events by the presence of a reciprocally connected GABAergic neuron.

No significant differences were found in the amplitude, charge, or decay time of the evoked EPSCs between homotypic glutamatergic and mixed pairs (Fig. 1), indicating specific regulation of glutamatergic spontaneous release by reciprocal GABAergic innervation. This form of plasticity is therefore different from the downregulation of both evoked and spontaneous glutamate release induced by prolonged cell depolarization (Moulder et al., 2004, 2006). Specific regulation of spontaneous release has been widely studied in recent years (for review, see Sutton and Schuman, 2009; Ramirez and Kavalali, 2011), but here we tie this to reciprocal innervation between GABAergic and glutamatergic neurons, an arrangement resembling the feedback inhibition loops between pyramidal neurons and various types of basket cells in the hippocampus (Kullmann, 2011). Specific regulation of spontaneous release might be realized using synapses supporting spontaneous release more than evoked release (Atasoy et al., 2008); changes in basal $\left[\mathrm{Ca}^{2+}\right]_{\mathrm{i}}$, which will affect spontaneous more than evoked release (Lou et al., 2005; Sun et al., 2007); separate vesicle pools for spontaneous release (Sara et al., 2005; Fredj and Burrone, 2009), which is controversial (Hua et al., 2010); or by changing the expression level of proteins specifically promoting spontaneous release, such as Doc2 (Groffen et al., 2010; Pang et al., 2011) and vtila (Ramirez et al., 2012).

Interestingly, the change in mEPSC frequency was not restricted to interneuronal synaptic contacts, but was extended to autaptic connections formed by the glutamatergic neuron onto itself, indicating global changes to the glutamatergic neuron. Such global changes could come about developmentally as a result of the different expression of transcriptional regulators, such as methyl-CpG-binding proteins, which are known to specifically regulate spontaneous release (Nelson et al., 2008). Reciprocal innervation might change the firing pattern of the neuron, which could lead to global changes. However, long-term application of TTX did not prevent the decrease in mEPSC frequency (Fig. 3D), arguing against this explanation. Therefore, the reduction in mEPSC frequency probably results from spontaneous GABA release and $\mathrm{GABA}_{\mathrm{A}}$ activation, consistent with the negative correlation between $\mathrm{mEPSC}$ and MIPSC frequency within mixed pairs (Fig. 5C,D). The mechanism might involve a different basal calcium homeostasis, since incubation with BAPTA-AM removed the difference in mEPSC frequencies between homotypic and mixed pairs (Fig. $6 \mathrm{C}$, control mixed pairs). A possible mechanism is that stabilization of the membrane potential by spontaneous GABA release will reduce calcium influx and lead to a lower resting $\left[\mathrm{Ca}^{2+}\right]_{\text {i. }}$ Since acute application of bicuculline did not change mEPSC frequency, and because the disinhibition of mEPSC in the synaptotagmin-1-null neurons was not found in homotypic glutamatergic pairs even after BAPTA-AM treatment, the difference between homotypic and mixed pairs must be consolidated developmentally through a differently organized release machinery. 
Our finding might be relevant for the previously reported lower mEPSC rate in organotypic or acute hippocampal slices compared with dissociated cultures (Cingolani and Goda, 2008; Pozo and Goda, 2010). In situ, most or all glutamatergic neurons partake in organized feedback loops with GABAergic neurons, whereas in dissociated cultures connections are random and survival of GABAergic neurons variable. Using astrocyte islands with exactly two reciprocally connected neurons, we create a situation where every glutamatergic neuron experiences a similar and supposedly strong GABAergic innervation. This preparation might prove useful for studying the developmental and molecular changes happening specifically as a function of feedback inhibition.

Knock-out studies established synaptotagmin-1 as the essential $\mathrm{Ca}^{2+}$ sensor for fast synchronous release (Geppert et al., 1994; Voets et al., 2001; Yoshihara and Littleton, 2002; Nishiki and Augustine, 2004), but controversies persist as to whether and how synaptotagmin-1 affects spontaneous release. Synaptotagmin-1 or -2 clamps spontaneous release in slices and mixed cultures (Broadie et al., 1994; Littleton et al., 1994; Pang et al., 2006; Chicka et al., 2008; Kerr et al., 2008; Kochubey and Schneggenburger, 2011), but not in autaptic neurons (Geppert et al., 1994; Liu et al., 2009) or in glutamatergic pairs (Fig. 5). Liu et al. (2009) observed unclamping of spontaneous release in synaptotagmin-1-null mutant micronetworks containing three or more neurons. Those experiments were probably performed on cultures containing mostly glutamatergic neurons. An increased number of glutamatergic neurons-and therefore synapses-might make it progressively easier to detect small increases in mEPSC frequencies upon synaptotagmin-1 deletion, which might already be present in autaptic neurons or glutamatergic pairs without reaching significance. In addition, with an increased number of neurons, the probability of including a GABAergic neuron in the network increases; whether this played a role in the study by Liu et al. (2009) remains unknown. Our data show that within neuronal pairs the unclamping of spontaneous release can already be dominant, but only in glutamatergic neurons forming reciprocal connections with a GABAergic neuron (Fig. 5B2). Thus, the presence of a GABAergic neuron plays a distinct role in setting the mEPSC frequency in wildtype and synaptotagmin-1-null neurons.

The majority of miniature release events are driven by $\left[\mathrm{Ca}^{2+}\right]_{\mathrm{i}}$ (Xu et al., 2009; Yang et al., 2010; Williams et al., 2012), implying that differences in $\left[\mathrm{Ca}^{2+}\right]_{\mathrm{i}}$ homeostasis, $\mathrm{Ca}^{2+}$ influx or $\mathrm{Ca}^{2+}$ release from intracellular stores (Emptage et al., 2001) might cause differences in miniature frequency (for a different view regarding mEPSCs, see Vyleta and Smith, 2011). However, the difference in mEPSC frequency between WT and synaptotagmin1-null mixed pairs was preserved in the presence of BAPTA, whereas in glutamatergic pairs the frequency in BAPTA was no different after elimination of synaptotagmin-1. This points to a different organization of the basal release machinery, such that spontaneous release is driven by machinery that is partly independent of synaptotagmin-1 in glutamatergic pairs and autaptic neurons. In contrast, in mixed cultures the majority of miniature release is driven by calcium binding to synaptotagmin-1 (Xu et al., 2009), indicating that synaptotagmin-1 exerts a strong clamp on miniature release. Overall, our data on the synaptotagmin-1null neurons demonstrate that a phenotype similar to that found in mixed cultures can be obtained using only two reciprocally connected neurons in vitro, verifying that such minimal networks reproduce an important feature of larger mixed cultures.
In conclusion, using neuronal pairs we have identified a role for reciprocal GABAergic/glutamatergic innervation in downregulating mEPSC frequency. It remains to be seen whether such a mechanism is involved in organizing the much more complex network of the intact brain. It appears likely that the effect is mediated by mIPSCs and translated by the membrane potential into a difference in calcium homeostasis, which in turn changes the organization of the release machinery. We note that glutamatergic neurons in situ initially develop in isolation until the formation of functional contacts leading to feedforward and feedback innervation, and that synapses pass through a developmental phase characterized by spontaneous, but no evoked release (Mozhayeva et al., 2002). Thus, the higher mEPSC rate in isolated glutamatergic neurons might be a hallmark of an immature glutamatergic neuron (Liu et al., 2013) and serve as a signal that a developing glutamatergic neuron is not yet under GABAergic feedback control.

\section{References}

Aoto J, Nam CI, Poon MM, Ting P, Chen L (2008) Synaptic signaling by all-trans retinoic acid in homeostatic synaptic plasticity. Neuron 60:308320. CrossRef Medline

Atasoy D, Ertunc M, Moulder KL, Blackwell J, Chung C, Su J, Kavalali ET (2008) Spontaneous and evoked glutamate release activates two populations of NMDA receptors with limited overlap. J Neurosci 28:1015110166. CrossRef Medline

Bekkers JM, Stevens CF (1991) Excitatory and inhibitory autaptic currents in isolated hippocampal neurons maintained in cell culture. Proc Natl Acad Sci U S A 88:7834-7838. CrossRef Medline

Broadie K, Bellen HJ, DiAntonio A, Littleton JT, Schwarz TL (1994) Absence of synaptotagmin disrupts excitation-secretion coupling during synaptic transmission. Proc Natl Acad Sci U S A 91:10727-10731. CrossRef Medline

Carter AG, Regehr WG (2002) Quantal events shape cerebellar interneuron firing. Nat Neurosci 5:1309-1318. CrossRef Medline

Chicka MC, Hui E, Liu H, Chapman ER (2008) Synaptotagmin arrests the SNARE complex before triggering fast, efficient membrane fusion in response to Ca2+. Nat Struct Mol Biol 15:827-835. CrossRef Medline

Cingolani LA, Goda Y (2008) Differential involvement of beta3 integrin in pre- and postsynaptic forms of adaptation to chronic activity deprivation. Neuron Glia Biol 4:179-187. CrossRef Medline

Crawford DC, Jiang X, Taylor A, Moulder KL, Mennerick S (2012) Differential requirement for protein synthesis in presynaptic unmuting and muting in hippocampal glutamate terminals. PLoS One 7:e51930. CrossRef Medline

Emptage NJ, Reid CA, Fine A (2001) Calcium stores in hippocampal synaptic boutons mediate short-term plasticity, store-operated Ca2+ entry, and spontaneous transmitter release. Neuron 29:197-208. CrossRef Medline

Fredj NB, Burrone J (2009) A resting pool of vesicles is responsible for spontaneous vesicle fusion at the synapse. Nat Neurosci 12:751-758. CrossRef Medline

Geppert M, Goda Y, Hammer RE, Li C, Rosahl TW, Stevens CF, Südhof TC (1994) Synaptotagmin I: a major Ca2 + sensor for transmitter release at a central synapse. Cell 79:717-727. CrossRef Medline

Glitsch M (2006) Selective inhibition of spontaneous but not Ca2+ -dependent release machinery by presynaptic group II mGluRs in rat cerebellar slices. J Neurophysiol 96:86-96. CrossRef Medline

Granseth B, Odermatt B, Royle SJ, Lagnado L (2006) Clathrin-mediated endocytosis is the dominant mechanism of vesicle retrieval at hippocampal synapses. Neuron 51:773-786. CrossRef Medline

Groffen AJ, Martens S, Díez Arazola R, Cornelisse LN, Lozovaya N, de Jong AP, Goriounova NA, Habets RL, Takai Y, Borst JG, Brose N, McMahon HT, Verhage M (2010) Doc2b is a high-affinity Ca2+ sensor for spontaneous neurotransmitter release. Science 327:1614-1618. CrossRef Medline

Hua Y, Sinha R, Martineau M, Kahms M, Klingauf J (2010) A common origin of synaptic vesicles undergoing evoked and spontaneous fusion. Nat Neurosci 13:1451-1453. CrossRef Medline

Kerr AM, Reisinger E, Jonas P (2008) Differential dependence of phasic 
transmitter release on synaptotagmin 1 at GABAergic and glutamatergic hippocampal synapses. Proc Natl Acad Sci U S A 105:15581-15586. CrossRef Medline

Kochubey O, Schneggenburger R (2011) Synaptotagmin increases the dynamic range of synapses by driving $\mathrm{Ca}(2)+$-evoked release and by clamping a near-linear remaining $\mathrm{Ca}(2)+$ sensor. Neuron 69:736-748. CrossRef Medline

Kullmann DM (2011) Interneuron networks in the hippocampus. Curr Opin Neurobiol 21:709-716. CrossRef Medline

Littleton JT, Stern M, Perin M, Bellen HJ (1994) Calcium dependence of neurotransmitter release and rate of spontaneous vesicle fusions are altered in Drosophila synaptotagmin mutants. Proc Natl Acad Sci U S A 91:10888-10892. CrossRef Medline

Liu H, Dean C, Arthur CP, Dong M, Chapman ER (2009) Autapses and networks of hippocampal neurons exhibit distinct synaptic transmission phenotypes in the absence of synaptotagmin I. J Neurosci 29:7395-7403. CrossRef Medline

Liu H, Chapman ER, Dean C (2013) "Self” versus "non-self” connectivity dictates properties of synaptic transmission and plasticity. PLoS One 8:e62414. CrossRef Medline

Lou X, Scheuss V, Schneggenburger R (2005) Allosteric modulation of the presynaptic Ca2+ sensor for vesicle fusion. Nature 435:497-501. CrossRef Medline

Moulder KL, Meeks JP, Shute AA, Hamilton CK, de Erausquin G, Mennerick S (2004) Plastic elimination of functional glutamate release sites by depolarization. Neuron 42:423-435. CrossRef Medline

Moulder KL, Jiang X, Taylor AA, Olney JW, Mennerick S (2006) Physiological activity depresses synaptic function through an effect on vesicle priming. J Neurosci 26:6618-6626. CrossRef Medline

Mozhayeva MG, Sara Y, Liu X, Kavalali ET (2002) Development of vesicle pools during maturation of hippocampal synapses. J Neurosci 22:654665. Medline

Naldini L, Blömer U, Gallay P, Ory D, Mulligan R, Gage FH, Verma IM, Trono D (1996) In vivo gene delivery and stable transduction of nondividing cells by a lentiviral vector. Science 272:263-267. CrossRef Medline

Nelson ED, Kavalali ET, Monteggia LM (2008) Activity-dependent suppression of miniature neurotransmission through the regulation of DNA methylation. J Neurosci 28:395-406. CrossRef Medline

Nishiki T, Augustine GJ (2004) Synaptotagmin I synchronizes transmitter release in mouse hippocampal neurons. J Neurosci 24:6127-6132. CrossRef Medline

Nosyreva E, Kavalali ET (2010) Activity-dependent augmentation of spontaneous neurotransmission during endoplasmic reticulum stress. J Neurosci 30:7358-7368. CrossRef Medline

Pang ZP, Sun J, Rizo J, Maximov A, Südhof TC (2006) Genetic analysis of synaptotagmin 2 in spontaneous and $\mathrm{Ca} 2+$-triggered neurotransmitter release. EMBO J 25:2039-2050. CrossRef Medline

Pang ZP, Bacaj T, Yang X, Zhou P, Xu W, Südhof TC (2011) Doc2 supports spontaneous synaptic transmission by a $\mathrm{Ca}(2+)$-independent mechanism. Neuron 70:244-251. CrossRef Medline

Pozo K, Goda Y (2010) Unraveling mechanisms of homeostatic synaptic plasticity. Neuron 66:337-351. CrossRef Medline

Ramirez DM, Kavalali ET (2011) Differential regulation of spontaneous and evoked neurotransmitter release at central synapses. Curr Opin Neurobiol 21:275-282. CrossRef Medline

Ramirez DM, Khvotchev M, Trauterman B, Kavalali ET (2012) Vtila iden- tifies a vesicle pool that preferentially recycles at rest and maintains spontaneous neurotransmission. Neuron 73:121-134. CrossRef Medline

Sara Y, Virmani T, Deák F, Liu X, Kavalali ET (2005) An isolated pool of vesicles recycles at rest and drives spontaneous neurotransmission. Neuron 45:563-573. CrossRef Medline

Schmitz SK, Hjorth JJ, Joemai RM, Wijntjes R, Eijgenraam S, de Bruijn P, Georgiou C, de Jong AP, van Ooyen A, Verhage M, Cornelisse LN, Toonen RF, Veldkamp W (2011) Automated analysis of neuronal morphology, synapse number and synaptic recruitment. J Neurosci Methods 195:185-193. CrossRef Medline

Shen W, Wu B, Zhang Z, Dou Y, Rao ZR, Chen YR, Duan S (2006) Activityinduced rapid synaptic maturation mediated by presynaptic cdc 42 signaling. Neuron 50:401-414. CrossRef Medline

Sun J, Pang ZP, Qin D, Fahim AT, Adachi R, Südhof TC (2007) A dual$\mathrm{Ca} 2+$-sensor model for neurotransmitter release in a central synapse. Nature 450:676-682. CrossRef Medline

Sutton MA, Schuman EM (2009) Partitioning the synaptic landscape: distinct microdomains for spontaneous and spike-triggered neurotransmission. Sci Signal 2:pe19. CrossRef Medline

Sutton MA, Ito HT, Cressy P, Kempf C, Woo JC, Schuman EM (2006) Miniature neurotransmission stabilizes synaptic function via tonic suppression of local dendritic protein synthesis. Cell 125:785-799. CrossRef Medline

Sutton MA, Taylor AM, Ito HT, Pham A, Schuman EM (2007) Postsynaptic decoding of neural activity: eEF2 as a biochemical sensor coupling miniature synaptic transmission to local protein synthesis. Neuron 55:648661. CrossRef Medline

Toonen RF, Wierda K, Sons MS, de Wit H, Cornelisse LN, Brussaard A, Plomp JJ, Verhage M (2006) Munc18-1 expression levels control synapse recovery by regulating readily releasable pool size. Proc Natl Acad Sci U S A 103:18332-18337. CrossRef Medline

Voets T, Moser T, Lund PE, Chow RH, Geppert M, Südhof TC, Neher E (2001) Intracellular calcium dependence of large dense-core vesicle exocytosis in the absence of synaptotagmin I. Proc Natl Acad Sci U S A 98: 11680-11685. CrossRef Medline

Vyleta NP, Smith SM (2011) Spontaneous glutamate release is independent of calcium influx and tonically activated by the calcium-sensing receptor. J Neurosci 31:4593-4606. CrossRef Medline

Wierenga CJ, Walsh MF, Turrigiano GG (2006) Temporal regulation of the expression locus of homeostatic plasticity. J Neurophysiol 96:2127-2133. CrossRef Medline

Wilcox KS, Buchhalter J, Dichter MA (1994) Properties of inhibitory and excitatory synapses between hippocampal neurons in very low density cultures. Synapse 18:128-151. CrossRef Medline

Williams C, Chen W, Lee CH, Yaeger D, Vyleta NP, Smith SM (2012) Coactivation of multiple tightly coupled calcium channels triggers spontaneous release of GABA. Nat Neurosci 15:1195-1197. CrossRef Medline

Xu J, Pang ZP, Shin OH, Südhof TC (2009) Synaptotagmin-1 functions as a $\mathrm{Ca} 2+$ sensor for spontaneous release. Nat Neurosci 12:759-766. CrossRef Medline

Yang X, Kaeser-Woo YJ, Pang ZP, Xu W, Südhof TC (2010) Complexin clamps asynchronous release by blocking a secondary $\mathrm{Ca}(2+)$ sensor via its accessory alpha helix. Neuron 68:907-920. CrossRef Medline

Yoshihara M, Littleton JT (2002) Synaptotagmin I functions as a calcium sensor to synchronize neurotransmitter release. Neuron 36:897-908. Medline 